\title{
ARTICLE \\ Carsharing-facilitating neighbourhood choice: a mixed logit model
}

\author{
Juan Wang ${ }^{1}$ (D) $\cdot$ Gamze Z. Dane ${ }^{1} \cdot$ Harry J. P. Timmermans ${ }^{2,3}$
}

Received: 11 August 2019 / Accepted: 8 October 2020 / Published online: 20 October 2020

(c) The Author(s) 2020

\begin{abstract}
Carsharing-facilitating neighbourhood is an emerging concept in urban development that combines carsharing, sustainable transportation-residential planning, and attractive housing to reduce private car use and improve neighbourhood quality. To investigate residents' preferences for such neighbourhoods, a stated choice experiment was designed that systematically varied attributes of carsharing-facilitating neighbourhoods to elicit their utility for people with a particular socio-demographic profile. The survey was conducted among residents who currently live in densely populated urban areas in The Netherlands. In total, 610 valid responses were obtained for analysis. To derive the utility of carsharing-facilitating neighbourhoods of a particular profile, a mixed logit model was estimated. Results indicate that the utility of a carsharing-facilitating neighbourhood primarily depends on carsharing cost, required carsharing booking time, green space density, housing costs, housing type, housing size and housing building year. Besides, the utility varies with socio-demographic characteristics, such as age, household composition, net monthly income, work status and education level. The estimated results can help policymakers and real estate developers understand the contribution of particular factors to the utility of carsharing-facilitating neighbourhoods, and determine target groups to develop implementation strategies.
\end{abstract}

Keywords Carsharing-facilitating neighbourhood $\cdot$ Mixed logit model $\cdot$ Stated choice experiment

Juan Wang

J.Wang.2@tue.nl

Gamze Z. Dane

g.z.dane@ tue.nl

Harry J. P. Timmermans

H.J.P.Timmermans@tue.nl

1 Department of the Built Environment, Eindhoven University of Technology, P.O. Box 513,

5600 MB Eindhoven, Netherlands

2 Department of the Built Environment, Eindhoven University of Technology, Eindhoven, Netherlands

3 Nanjing University of Aeronautics and Astronautics, Nanjing, China 


\section{Introduction}

Due to increasing private car ownership, cities are facing major challenges such as heavy parking pressure, congestion and air pollution (e.g., Banister et al. 2000; Newman et al. 2016). Negative effects of private car ownership are also visible at the neighbourhood scale. For example, increasingly more public space is used for parking, which reduces the availability of neighbourhood green/open space (e.g., Wiersma et al. 2016). Similarly, road-side parking in neighbourhoods increases risks to residents (e.g., Biswas et al. 2017). In response, urban planners, transport operators and residents began to rethink how to deal with private car ownership and the problems it brings through transportation and land use planning (e.g., Chatman 2009; De Vos et al. 2012).

The idea to influence travel behaviors through urban design concepts is not new. The concept of car-free neighbourhood is an early attempt to reduce residents' private car use and improve neighbourhood quality. By integrating green transportation (e.g., walking and cycling, convenient public transport), low parking norms to restrict private cars, and sustainable residential planning (e.g., high green space density, safe children playgrounds, green architecture design etc.), these neighbourhoods attempted to reduce private car-ownership and improved neighbourhood quality (e.g., Kushner 2005; Foletta and Field 2011). To note, though these neighbourhoods were called car-free neighbourhoods, but most were not truly car-free but rather neighbourhoods with relatively low parking norms. Meanwhile carsharing was sometimes an element of the design, but the number of available cars was typically very small (one shared-car for every 50-500 households) and making reservation took quite some effort (e.g., Kushner 2005). Since the 1990s, several of these car-free neighbourhoods have been developed in Germany, UK, Sweden, Austria and the Netherlands. Examples include Vauban (1992), Stellwerk 60 (2011), Greenwich Millennium Village (1999), Hammarby Sjostad (ongoing) and GWL Terrein (1995) etc. However, the concept diffused rather slowly. Kushner (2005) and Morris et al. (2009) argued that to promote the large development of car-free neighbourhoods, more incentives, such as the provisions of more diverse urban-scale green mobility options are needed.

From a transportation planning perspective, the concept of carsharing has recently been matured. The general availability of smartphones and digital platforms represented a huge step forward in reducing consumer burden in information acquisition, booking and paying for shared-cars. Moreover, advanced algorithms allow the optimal (re)distribution of shared-cars in time and space (Agatz et al. 2012). Most carsharing schemes are based on neighbourhoods, i.e. target individual customers in a particular residential area (e.g., Millard-Ball et al. 2005; Shaheen and Cohen 2007). By paying a flexible fee for shared-car usage which covers the costs of vehicle use, insurance, maintenance and fuel, residents pay as they use shared-cars instead of facing the fixed costs which usually come with traditional car ownership (e.g. Rodier and Shaheen 2003; Coates 2013: European Commission 2017). As a supplement to the sustainable transport modes, carsharing indirectly promotes more walking, cycling, public transport use and reduces private car ownership (Loose 2015). Since the 1990s, but particularly over the last five to ten years, carsharing experienced substantial growth, especially in urban areas. According to Loose (2015), between 2006 and 2014 carsharing memberships have increased from 0.2 million to 2.2 million in Europe. Inspired by a growing carsharing market and the tendency to explore applications of platform technology, developers and municipal governments have started to incorporate carsharing as a major impetus into car-free neighbourhood developments to promote sustainability and improve neighbourhood quality, e.g., Merwede (Utrecht, 
the Netherlands) where one shared-car is planned for every three homes (Herranz 2020). We call these latest developments carsharing-facilitating neighbourhoods.

Carsharing-facilitating neighbourhood can be defined as a scheme orchestrated by local governments/planning authorities, typically in the context of public-private partnerships to combine carsharing, sustainable transportation-residential planning, and traditional attractive housing elements to reduce private car ownership and improve neighbourhood environment. This concept has been fuelled by the recent intensification of the climate change discussion and believed that the revamped concept may appeal to particular market segments due to changing attitudes, lifestyles and priorities. There are similarities and differences between the concepts carsharing-facilitating neighbourhood, car-free neighbourhood and carsharing. Carsharing-facilitating neighbourhood shares notions of sustainable transportation and residential planning implemented in traditional car-free neighbourhoods. A carsharing-facilitating neighbourhood also is a comprehensive housing/neighbourhood design concept which emphasizes green transportation planning and neighbourhood environment improvement. The key notion of the concept is that reduced parking infrastructure allows planners to develop more greenspace, safer children playgrounds and/or larger residential buildings to provide residents with better living environments and higher quality of life. However, in carsharing-facilitating neighbourhoods, carsharing serves a more prominent role. It is centrally offered as a service by the management office of the neighbourhood, while platform technology guarantees easy access to the service. This makes carsharing-facilitating neighbourhoods distinct from traditional car-free neighbourhoods where carsharing was only a tiny component.

The concept carsharing-facilitating neighbourhood is rather new. Projects known are still under discussion and research has been rare. The idea is not without risk and requires to enhance the feasibility and identify potentially interested market segments to convince stakeholders to invest in the developments. Thus in this study, we aim to investigate people's preferences and acceptance for carsharing-facilitating neighbourhoods, and the influences of sociodemographic variables on that. To that end, a stated choice experiment was applied, which is the best way to study preferences and acceptance in this particular context. Because the envisioned carsharing-facilitating neighbourhoods are not in full operation, it is impossible to conduct traditional surveys in these neighbourhoods to learn residential and transport preferences choices. Moreover, such surveys would introduce sample selection bias (e.g., Louviere et al. 2000). Although stated choice experiments are not perfect either, they do have a long history in residential preference research (e.g., Dieleman 1996; van de Vyvere et al. 1998; Kim et al. 2005), and their advantages in eliciting preferences have been well documented in many different choice domains (e.g., Louviere and Timmermans 1990; Louviere et al. 2000; Hensher et al. 2005), including carsharing (e.g., Kim et al. 2017).

The remainder of this paper is structured as follows. First, we briefly review existing research related to carsharing-facilitating neighbourhoods, such as carsharing, car-free neighbourhood and residential housing choice. Next, we describe the methodology used in our study. After that, the results of the model estimation are presented. Finally, we conclude the paper and discuss the limitations and directions of future research.

\section{Literature review}

To date, known carsharing-facilitating neighbourhood projects are at the planning stage. One of the most reported projects is Merwede (Utrecht, the Netherlands). This project plans to cover 24 hectares, providing 2000 residential units with diverse size, type etc. This 
project applies traditional car-free planning strategies (e.g., walking and cycling network construction, high green space density, mixed land use etc.), in addition widely available carsharing on site-one shared-car for every three homes (Herranz 2020). Another example is the renovation scheme of Strijp-s (Eindhoven, the Netherlands), which planes to ingrate carsharing as a major drive to reduce residents' private car ownership. As a result, reduced parking can be used for more greenspace to improve neighbourhood environment.

Since research specifically targeted at carsharing-facilitating neighbourhood is very limited, this literature review will focus on existing research related to the three ingredients of carsharing-facilitating neighbourhood, namely carsharing, car-free neighbourhood transportation-residential planning, and housing choice. The aim of this literature review is to identify the attributes influencing carsharing participation, car-free neighbourhoods design, and housing choice. The results will be used to design the stated choice experiment for carsharing-facilitating neighbourhood.

\subsection{Carsharing influential factors}

Sufficient research has found that pricing, accessibility, time-saving, vehicle diversities have significant influence on residents' acceptance of carsharing schemes. Lower pricing can significantly increase residents' willingness to join a carsharing program (e.g. Kim et al. 2005; Kato et al. 2013). By using stated-choice (SC) data, Kim et al. (2017) further found that reducing deposits, monthly membership fee and/or hourly rates is more effective in attracting new carsharing users than reducing distance-based rates or fuel cost. Second, high accessibility of shared-car parking is welcome by residents. A less than 5 min walking to shared-car parking is preferred most by users (e.g. Kim et al. 2005; Kato et al. 2013; Ciari et al. 2016; Wang and Yan 2016). Moreover, residents who are under severe parking pressures in their living neighbourhoods or cities incline to dedicated shared-car parking spaces (e.g. Kopp et al. 2015; Dowling and Kent 2015; Cartenì et al. 2016). Timesaving (time saved through using shared-cars, for example, shorter waiting time compared with using other transportation modes (Bock et al. 2005)) is another important attribute for users to subscribe to carsharing (e.g., Cartenì et al. 2016; Jae-Hun Joo 2017). By doing a stated choice experiment in South Korea among Socar ${ }^{1}$ users, Jae-Hun Joo (2017) found time-saving is even more important than cost-saving and social value. ${ }^{2}$ Last but not least, certain residents prefer a mix of shared vehicle types as they could cater their occasional demands, such as cargo moving etc. (e.g. Martens et al. 2015; Becker et al. 2017).

\subsection{Car-free neighbourhood planning}

Kushner (2005), Morris et al. (2009) and Foletta and Field (2011) reported case studies of existing well-known car-free neighbourhoods, such as Woltmannweg (Berlin), Vauban (Freiburg), Florisdorf (Vienna), GWL-Terrein (Amsterdam) and Slateford Green (Edinburgh). According to these authors, five principles were applied in car-free neighbourhood planning.

First, provide direct, safe and comfortable walking and cycling facilities and routes to give walking and cycling a competitive advantage over the cars. For example, build dense

\footnotetext{
1 Socar is the largest carsharing service in South Korea.

2 The degree of creating social and environmental value through carsharing (Joo 2017).
} 
walking and cycling networks; locate bicycle parking closer to home than car parking etc. Second, provide high-quality transit responsive to residents needs with high accessibility and diversity. For example, transit stops are planned within 500 meters to each home; bus/ tram frequencies are at least every $15 \mathrm{~min}$; low-cost travelling passes are available to keep using public transports affordable. Third, reduce parking supply ration. For example, residents were asked to contractually agree not to own cars; a small portion of residents can obtain parking space by competing in a lottery, or paying expensive costs for private parking. Fourth, create mixed land use. Housing, jobs, shops, leisure facilities etc. are ideally planned within walking distance. Fifth, provide more public space. Lands released by less private ownership and use are converted to more green or open space to improve neighbourhood environment.

\subsection{Residential housing choice}

Sufficient studies have indicated housing attributes, such as cost, size, type, location, and building age, commuting distance, public transportation network have significant influences on residents' housing choice. The influence of these attributes also varies with residents' socio-demographics which determine residents' housing needs and also the ability to pay a certain rent/mortgage (e.g., Dieleman 1996; de Palma et al. 2007; Olanrewaju and Woon 2017).

Housing prices have a major influence on residential choice. High prices usually decrease housing appeal as residents generally intended to minimize accommodation expense share of income (e.g., Srour et al. 2002; Zondag and Pieters 2005; de Palma et al. 2007; Zhou and Kockelman 2009). Single residents generally prefer smaller housing, while couples prefer larger living areas (Eliasson 2010). Residents' preferences to certain housing types significantly varies with marital status and local housing market (e.g., van de Vyvere et al. 1998; Habib and Miller 2009; Tu et al. 2017). For example, households with children prefer detached/semi-detached houses, while households without children prefer multi-family houses (e.g., Dieleman 1996; Axhausen et al. 2004). In the case of housing location, young households without children prefer to live in the city center where population density is higher and land use is mixed (e.g., Waddell 2006; Guo and Bhat 2007), while households with children tend to move away from the city center (Chen et al. 2008). The dislike of the city center was also found among high-income and the senior population (e.g., Weisbrod et al. 1980; Axhausen et al. 2004; Kim et al. 2005; Zondag and Pieters 2005; Pinjari et al. 2009; Schirmer et al. 2014). Besides, research found residents generally prefer housing built during 1960 and 1980 but dislike housing built before 1960 (e.g., van de Vyvere et al. 1998; Srour et al. 2002). In the case of commuting distance, Residents overall prefer shorter commuting distance-less than $30 \mathrm{~min}$ travelling (e.g., Srour et al. 2002). Good public transportation network and transit station accessibility can increase the housing appeal to residents especially who do not own private cars and/or commute by public transport (e.g., van de Vyvere et al. 1998; de Palma et al. 2005).

\section{Methodology}

In order to analyze residents' choice of carsharing-facilitating neighbourhoods, a stated choice experiment was constructed. Stated choice experiments combine attribute levels of choice alternatives according to the experiment design principles and request respondents 
(repeatedly) to choose an alternative they prefer from a set of choice sets. It assumed that respondents derive a utility from a bundle of attributes which describe the choice alternatives. Based on respondents' choices, part-worth utilities for attribute levels can be statistically estimated and how much weight respondents attach to different attributes and levels can be understood (Louviere et al. 2000). Stated choice experiments typically describe hypothetical decision contexts, which applies to our research-therefore carsharing-facilitating neighbourhood preferences.

The design of stated choice experiments starts with the elicitation of the attributes and attributes deemed to influence the choice behavior of interest. Based on the literature review and interviews with urban planners and real estate developers involved in carsharing-facilitating neighbourhoods, fifteen attributes were selected. As shown in Table 1, the selected attributes can be viewed as specifications of carsharing: carsharing costs, booking time, parking distance to home, parking distance to destination; specifications of the sustainable transportation-residential planning: commuting distance, public transport accessibility, housing location, private parking space, buildings/green area density, children playground safety; and specifications of housing: house type, ownership, size, price, building time. Each attribute takes on different levels. For example, carsharing booking time is assigned with four levels: " $\leq 10 \mathrm{~min}$ ", "11-20 min", "21-30 min", and ">30 min"; children playground safety is assigned with two levels: "improved safety of children playing area" and "present safety of children playing area".

The selected attributes and levels are then combined such that the carsharing-facilitating neighbourhood alternatives can be generated. However, because the number of alternatives generated by a full factorial design would be $4^{12} \times 2^{3}$ (twelve attributes take four levels and three attributes take two levels), which is too large to complete by respondents. We constructed an orthogonal fractional main effects design which reduces the alternatives number by satisfying the conditions of orthogonality attribute level balance and assuming a main effects only additive utility function (e.g., Hensher et al. 2005; Rao 2014). As a result of an orthogonal fractional design, 128 carsharing-facilitating neighbourhood alternatives were generated. To create the choice sets, each of the 128 alternatives serves as the first option and afterwards combined with the second option which was obtained by randomly sorting the generated 128 alternatives. Thus in total, 128 choice sets each of which contains two options were generated. Considering respondents may like neither sufficiently of the options or have no interest to live in a carsharing-facilitating neighbourhood, an "I like neither" option was added to each choice set. By applying a blocking procedure, the design was finally divided into 16 blocks, thus every respondent received 8 choice sets (e.g., Louviere et al. 2000). In each choice set, the respondents were asked to select their most preferred carsharing-facilitating neighbourhood alternative or the "I like neither" option.

To start the stated choice survey, respondents were given the following task instructions: "Increasing private car ownership has caused negative impacts on our environment. Carsharing, a sustainable transportation mode, is an efficient substitute for private cars, as it is environmental, convenient and economical. To protect the environment and save urban parking land, governments are proposing to construct/reconstruct carsharing-facilitating neighbourhoods to reduce private car ownership. Residents living in carsharing-facilitating neighbourhoods do not need to own cars but can be enrolled into to a carsharing program to use shared vehicles which are maintained by the carsharing organization with others in the neighbourhood at lower costs (residents are still allowed to own their private cars). Carsharing-facilitating neighbourhoods are designed with less parking space. To compensate, there will be more green space and/or larger houses. Imagine that you are considering choosing the residence. You will be given two residential options with different 


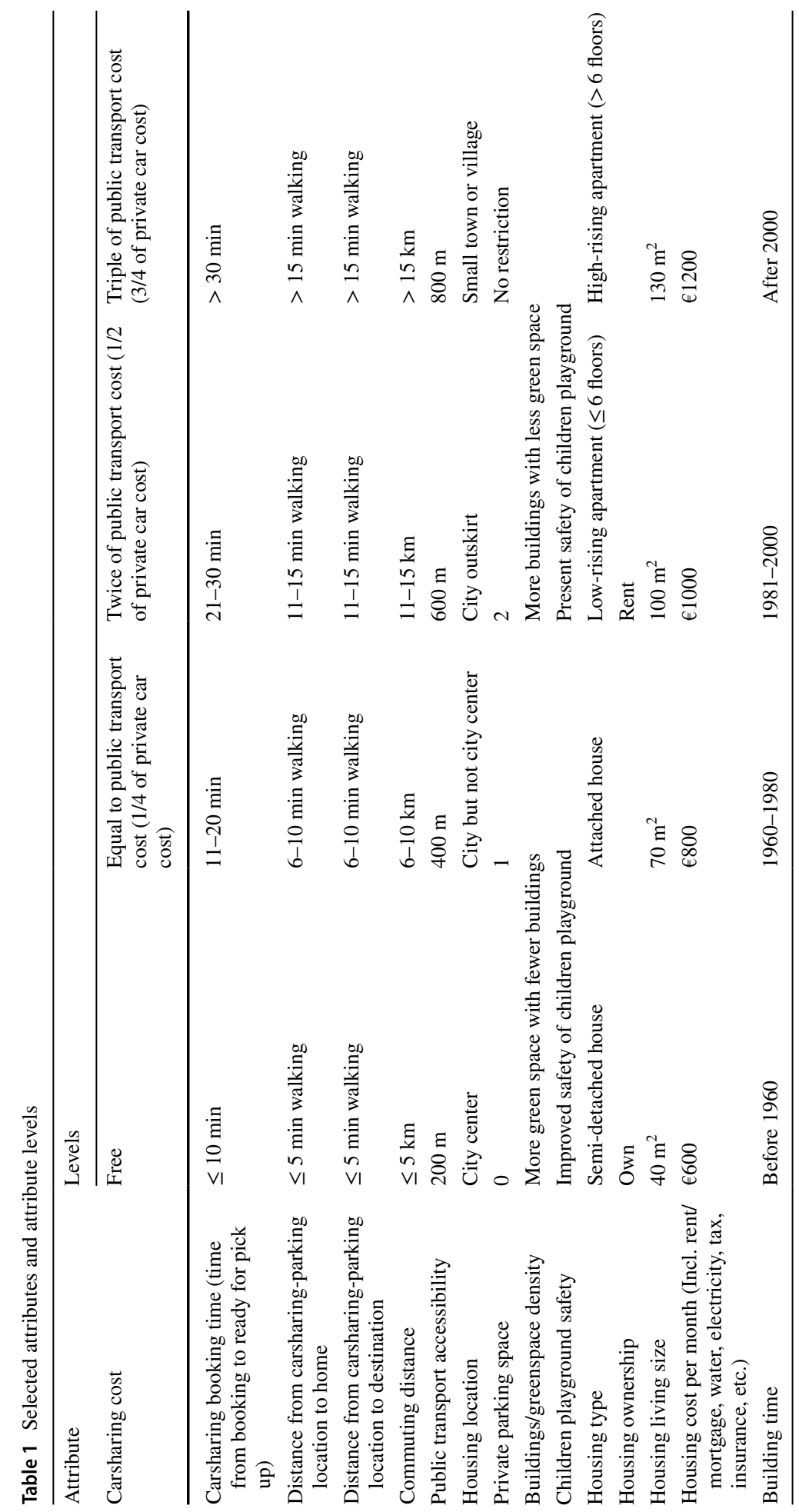


carsharing-facilitating neighbourhood characteristics. Please compare these options carefully considering the trade-off between different characteristics and indicate the option you would choose. If you don't like either sufficiently or you have no interest in living carsharing-facilitating neighbourhoods, just choose "I like neither" option.

\section{Results}

\subsection{Data collection}

The questionnaire used to collect the necessary data consisted of four parts: socio-demographic attributes, current housing built environment, travel behaviour and the stated choice experiment. It was administered online in September 2018 in densely populated cities (more than 1000 people $/ \mathrm{km}^{2}$ ) in the Netherlands. The reason we chose the densely populated cities is that existing research indicated residents in those cities have higher demands for car-free neighbourhood or carsharing because of the high parking costs and robust public transportation (e.g., Melia 2010; Nijland and van Meerkerk 2017). Thus, we suppose high-density cities are the potential carsharing-facilitating neighbourhood markets. In total, 12 cities were selected for the data collection, including large cities with a population larger than 300,000, such as Amsterdam, Rotterdam, The Hague, Utrecht; medium cities with a population between 200,000 and 300,000, such as Almere, Eindhoven and Groningen; and small cities with a population smaller than 200,000, such as Arnhem, Delft, Haarlem, Helmond and Leiden. ${ }^{3}$ The sample quota of the cities are proportional to their population. As a result of the data collection, 823 completed responses were obtained. After cleaning, 610 were used for analysis. The data was cleaned by omitting response with outliers or response completed in a very short time (it may indicate the respondents did not seriously read and consider the descriptions of the different profiles in the stated choice experiment).

\subsection{Sample characteristics}

An overview of the key sample characteristics is given in Table 2. Accordingly, $59.0 \%$ of the respondents are from large cities, $20.5 \%$ of the respondents are from medium cities, and the rest $20.5 \%$ of the respondents are from small cities. The sample is fairly evenly distributed across gender classes with $55.7 \%$ of the sample being male and $44.3 \%$ female. Age from 51 to 65 and older than 65 are the largest age groups with the shares of respectively $36.4 \%$ and $25.1 \%$. Young people aged between 18 and 35 is the smallest category. Regarding household composition, couple without children is the largest category $(32.3 \%)$ which is followed by singles without children (32.0\%), couples with children $(22.8 \%)$ and others $(12.9 \%)$. The largest net income category is less than $€ 1600$, accounting for $32.8 \%$, followed by the category $€ 1601$ to $€ 2400$ (29.2\%) and more than $€ 2400$ (20.3\%). $17.7 \%$ of the respondents chose not to tell the income. In the case of working status, $37.2 \%$ have fulltime jobs, $21.5 \%$ have part-time jobs, and $41.3 \%$ have retired or don't work. Vocational is the largest educational category (36.2\%), followed by HBO/Bachelor (27.4\%) and primary

\footnotetext{
3 The population statistics is from the website "UrbiStat AdminStat": https://ugeo.urbistat.com/AdminStat/ it/nl/demografia/dati-sintesi/paesi-bassi/528/1 and "CBS": https://www.cbs.nl/.
} 
Table 2 Sample socio-demographic characteristics

\begin{tabular}{llcc}
\hline & & Frequency & Percent (\%) \\
\hline Living city & Large city & 360 & 59.0 \\
& Medium city & 125 & 20.5 \\
Gender & Small city & 125 & 20.5 \\
& Male & 270 & 44.3 \\
Age & Female & 340 & 55.7 \\
& $18-35$ & 98 & 16.1 \\
& $36-50$ & 137 & 22.5 \\
Household composition & $51-65$ & 222 & 36.4 \\
& 65+ & 153 & 25.1 \\
& Couple, with children & 139 & 22.8 \\
Net Monthly Income & Couple, no children & 197 & 32.3 \\
& Single, no children & 195 & 32.0 \\
& Others & 79 & 12.9 \\
& $\leq € 1600$ & 200 & 32.8 \\
& $€ 1601-€ 2400$ & 178 & 29.2 \\
Work status & $>€ 2400$ & 124 & 20.3 \\
& I do not want to say. & 108 & 17.7 \\
Educational Level & Full-time & 227 & 37.2 \\
& Part-time & 131 & 21.5 \\
& Not working & 252 & 41.3 \\
& Primary and Secondary & 163 & 26.7 \\
& Vocational & 221 & 36.2 \\
& HBO/Bachelor & 167 & 27.4 \\
& Master or PhD & 59 & 9.7 \\
\hline
\end{tabular}

and secondary $(26.7 \%)$. Only $9.7 \%$ of the respondents have an educational degree as Master or $\mathrm{PhD}$.

\subsection{Estimation}

The model was assumed a linear additive utility function. The dependent variable is the respondents' choice of carsharing-facilitating neighbourhood. The independent variables are carsharing-facilitating neighbourhood attributes varied in the choice experiments and social-demographic characteristics of respondents. To specify the influence of each level of the attributes, effect coding was used, which means the estimated part-worth utilities sum to zero across the levels of one attribute. The likelihood ratio test statistic $[G 2=-2$ $(L L(0)-L L(B))]$ is used to test whether the estimated choice model $L L(B)$ significantly improves the null model $L L(0)$ (e.g., McFadden 1973; Buse 1982). McFadden's pseudo Rho-square $\left(\rho^{2}=1-L L(B) / L L(0)\right)$ is used to indicate the goodness of fit of the estimated choice model (McFadden 1973).

(1) Model fitness 
To start a discrete choice model estimation, multinomial logistic regression (MNL) was first applied as a based-model, which assumed all respondents in the choice tasks have the same preferences captured by the deterministic component of the utility function (e.g., Louviere et al. 2000). The MNL model resulted in a McFadden pseudo Rho-square of $0.112(<0.200)$, which indicated it failed in giving a decent fit and respondents may have heterogeneous preferences (e.g., McFadden 1973; Hensher et al. 2005).

Thus the mixed logit model was then estimated to capture the heterogeneity of individuals' propensity to live in carsharing-facilitating neighbourhoods. Normal distribution is assumed as we expect preferences to deviate from the mean in both directions equally, with most deviations around the mean, and more extreme deviations occurring less frequently (e.g., Greene 2001). For a good performance of random draws from the specified distribution, one thousand Halton sequences were used to estimate the parameters of the present mode (e.g., Train 2003; Bhat 2001). As a result, the McFadden pseudo Rho-square of estimated mixed logit model was obtained as $0.377(\geq 0.200)$, which shows a decent fit (e.g., McFadden 1973; Hensher et al. 2005). This result also proves that heterogeneity exists among individuals regarding the propensity to live in carsharing-facilitating neighbourhoods. The estimation results for the mixed logit model (ML) is showed in Table 3.

\section{(2) Main effects of carsharing-facilitating neighbourhood attributes}

The constant, entered as a random parameter, is negative but not significant, indicating that residents on average are less willing to live in carsharing-facilitating neighbourhoods. The significant standard deviation of the constant shows that there is intra and inter individual variation in the choices of respondents for carsharing-facilitating neighbourhoods.

To start with carsharing-related attributes, the estimated part-worth utility for carsharing cost compared to the cost of public transport and private car decreases rapidly with increasing carsharing cost. This result is in line with previous carsharing research findings that lower costs encourage residents to subscribe to carsharing services (e.g., MillardBall et al. 2005). According to the standard deviation of the assumed normally distributed random parameters, lower carsharing costs, such as free or equal to public transport cost are estimated with a large and significant standard deviation. This result suggests that the tastes of respondents for lower carsharing costs vary significantly across the samples. We infer that, for the residents who have no interest living in carsharing-facilitating neighbourhoods, even lower free carsharing costs are unattractive. In the case of carsharing booking time, utility tends to more or less monotonically decreasing with increasing booking time. Overall, the effects of carsharing booking time on utility are positive if the booking time is shorter than $20 \mathrm{~min}$. Interestingly, the estimated standard deviation is significantly high for the carsharing booking time between 20 and $30 \mathrm{~min}$. One possible reason is that, the carsharing-facilitating neighbourhood enthusiasts (e.g., people dedicated to environmental benefits) have higher tolerance with a longer carsharing booking time. In the case of carsharing parking distance to home, the estimated part-worth utility fluctuates with an increasing distance. As a result, the estimated effects of a short distance-less than $5 \mathrm{~min}$, or a relatively long distance-11 to 15 min walking distance are positive. According to previous research, carsharing users prefer a short shared-cars accessibility for convenience (e.g. Kim et al. 2005), however, car-free neighbourhood residents prefer no-car environment, thus neighbourhood periphery parking is preferred (e.g., Kushner 2005). Since carsharing-facilitating neighbourhood has the double features of carsharing and car-free neighbourhood, this estimated result is reasonable. The estimated utility of carsharing 


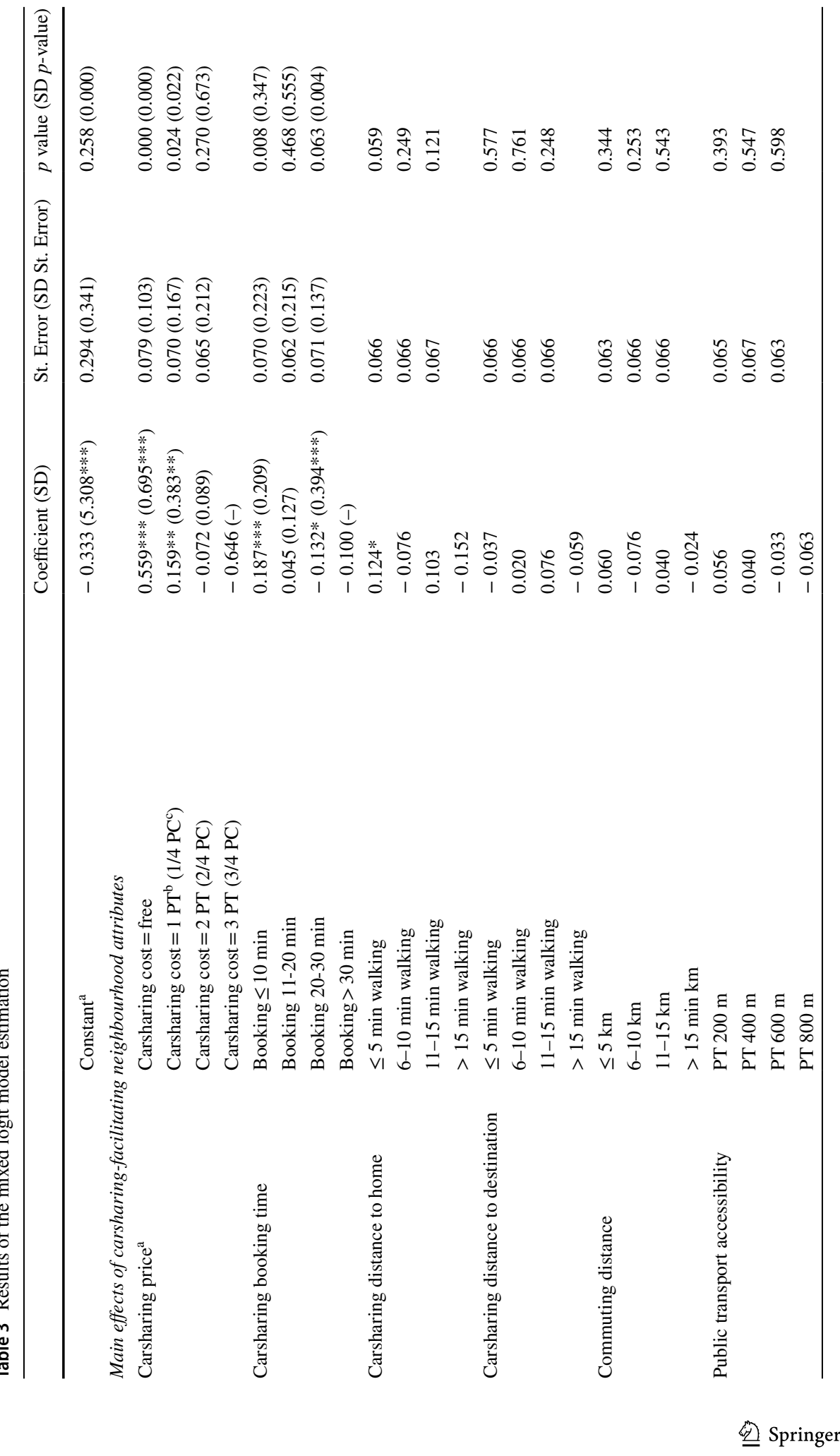




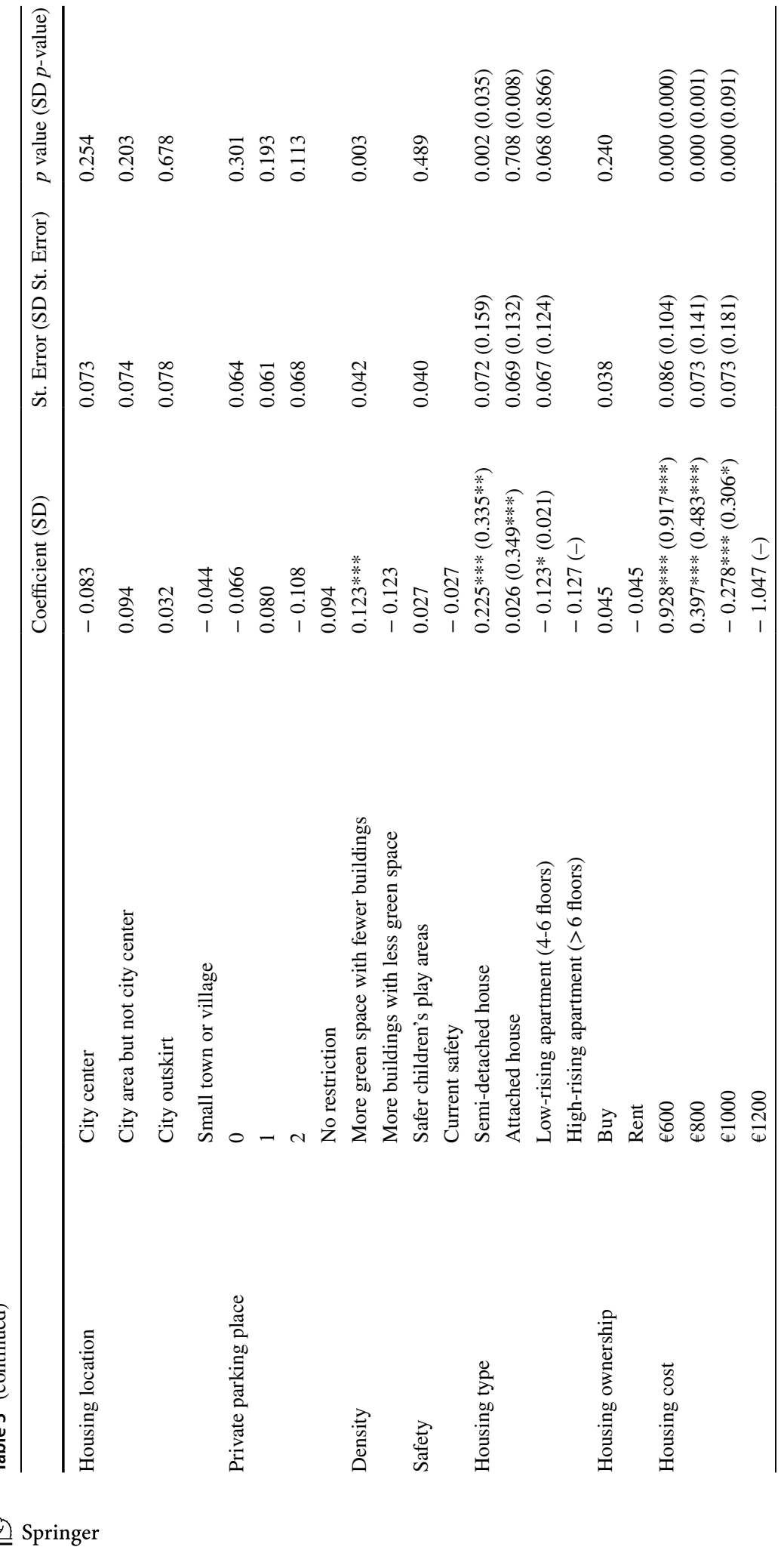




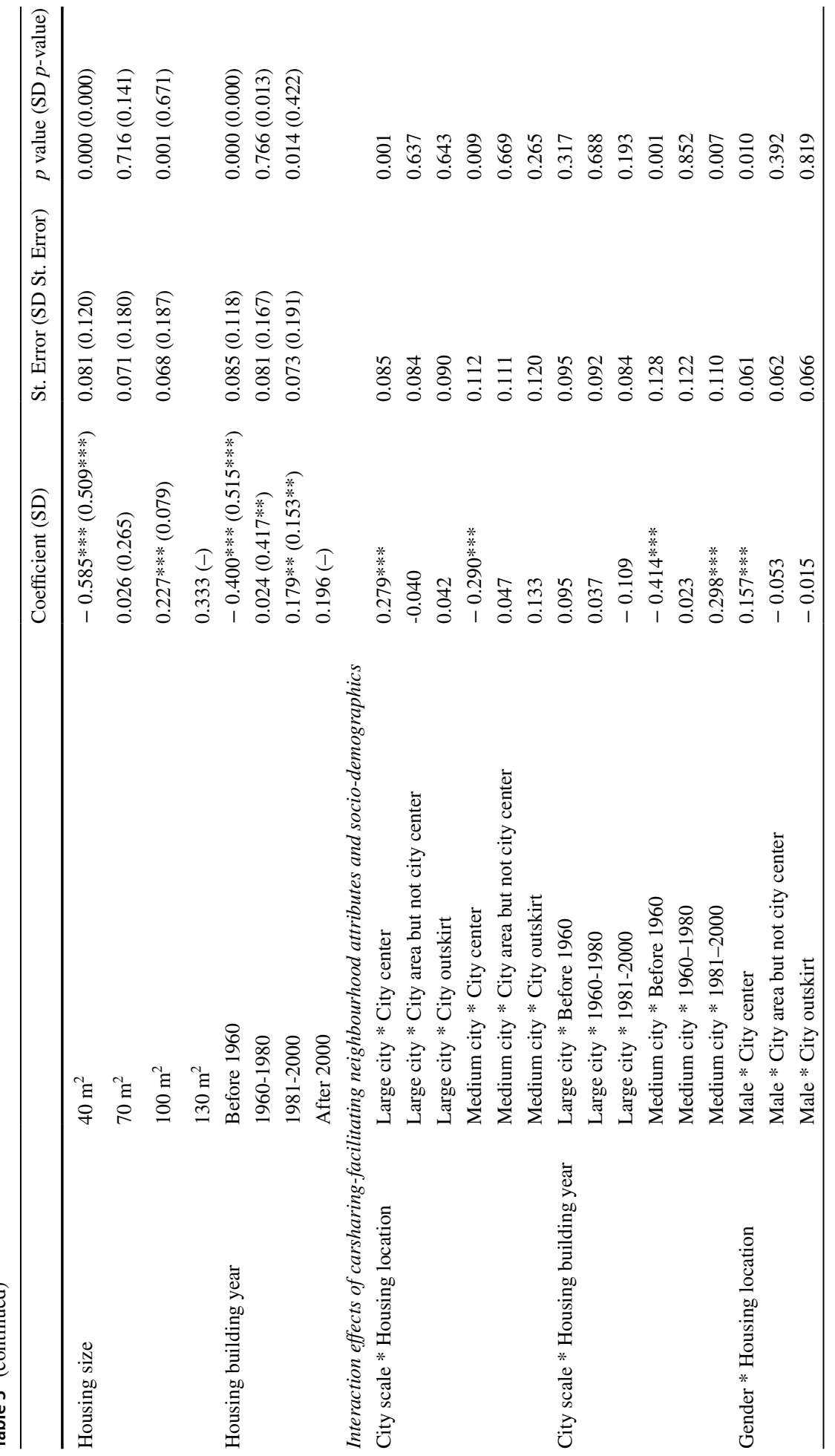




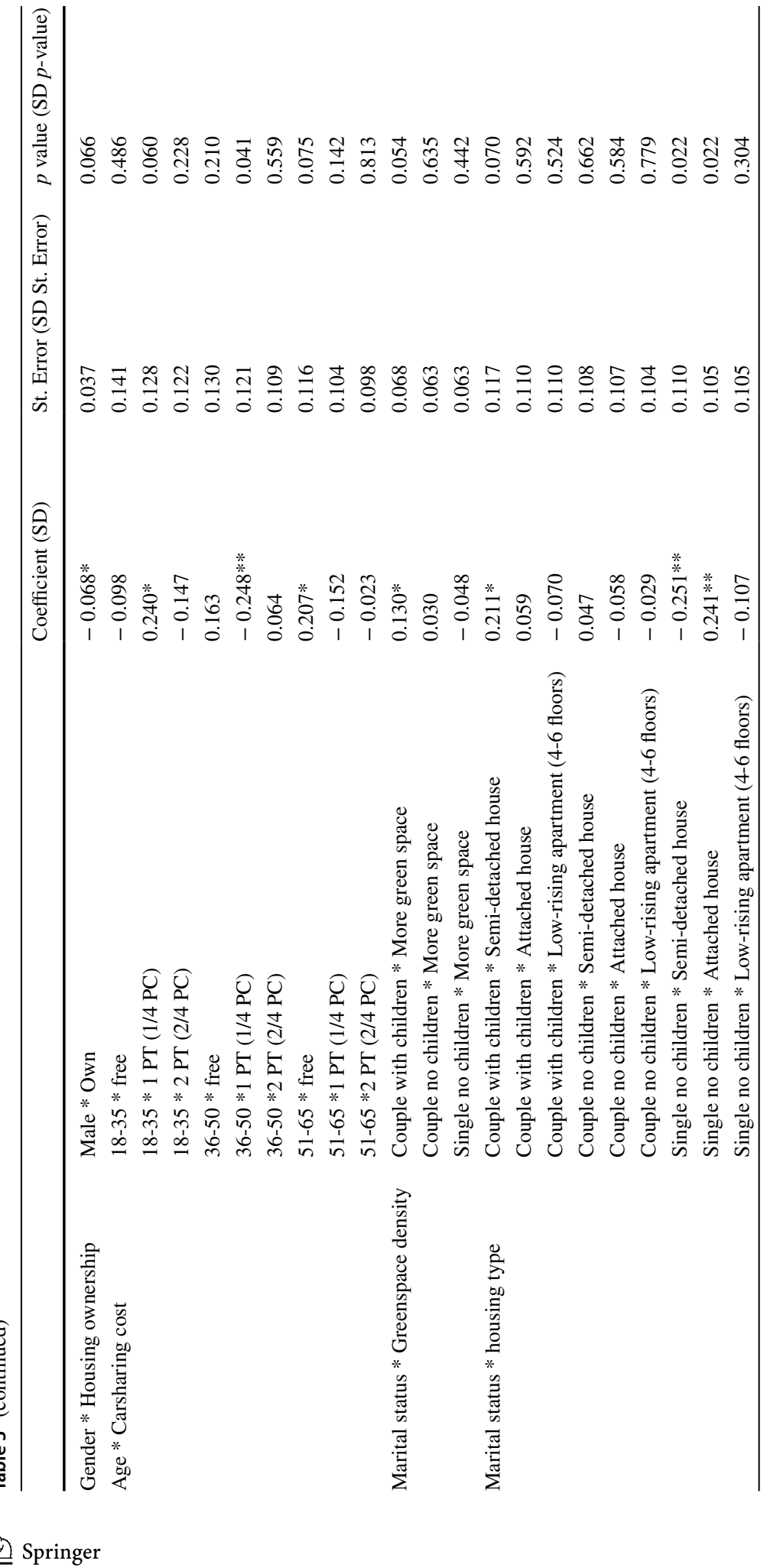




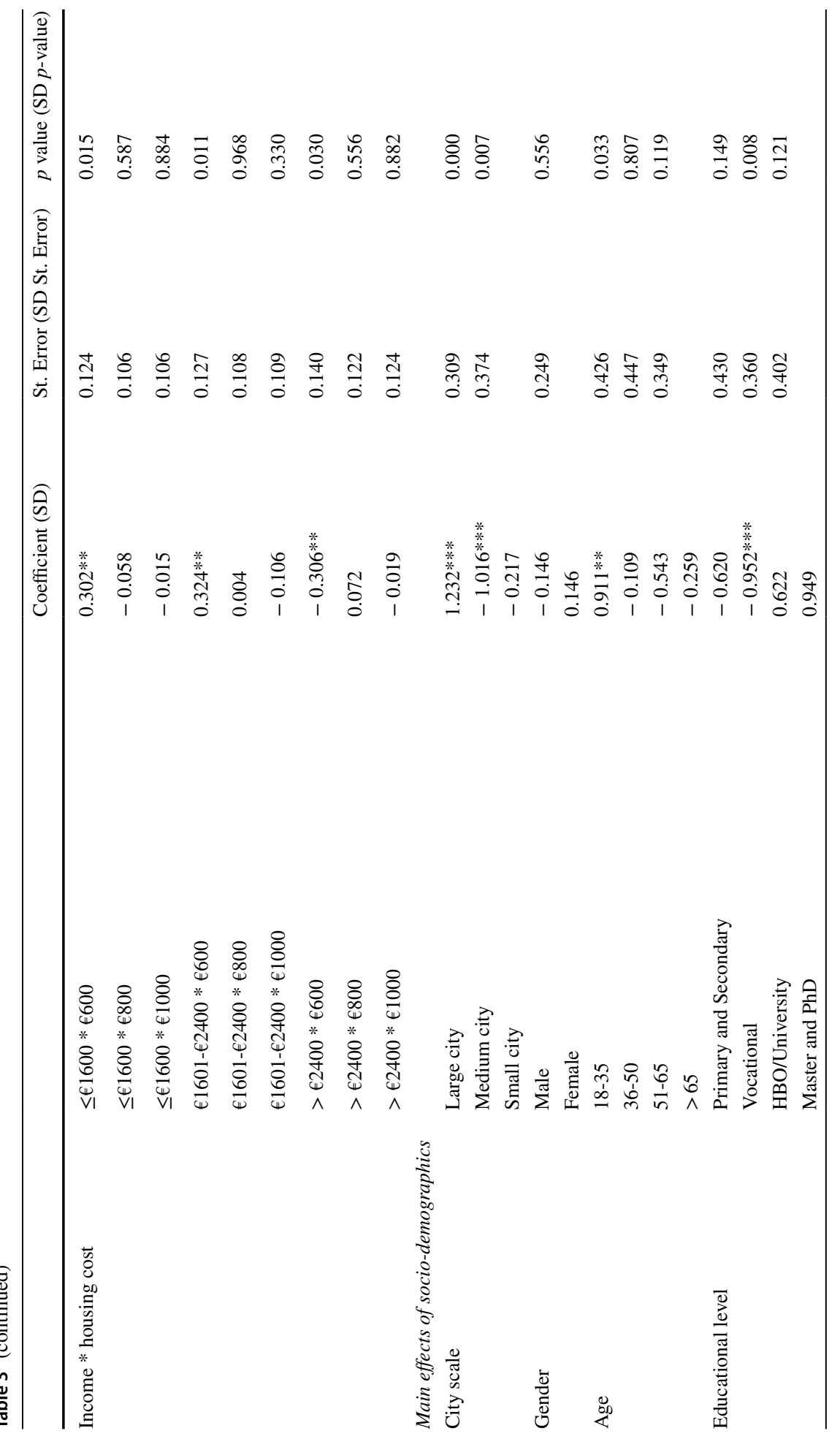




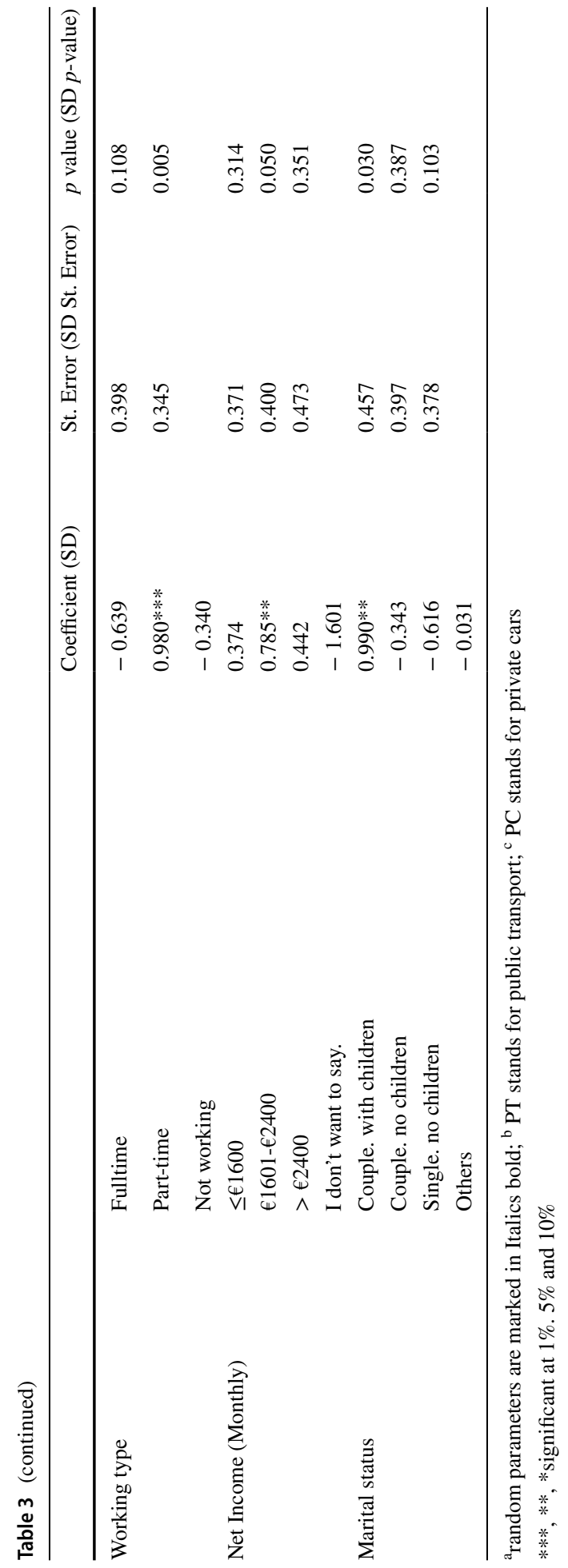

Springer 
parking distance to the destination first increases then decreases with an increasing distance. However, the effect is small and insignificant, thus not important for carsharingfacilitating neighbourhood choices.

Regarding the effects of sustainable transportation-residential land use planning attributes, the majority, except green space, are estimated with tiny and insignificant effects. This result may explain why traditional car-free neighbourhoods (mainly applied transportation-residential planning strategies) had limited appealing to residents (e.g., Kushner 2005). Regarding the estimated effects of green space density, the part-worth utility of high green space density is significantly positive. It indicates that more green space in carsharing-facilitating neighbourhood can attract residents. This result is in line with the previous finding that car-free neighbourhood residents highly complimented for dense green space in the neighbourhoods because it provides more natural space for children (e.g., Coates 2013).

The estimated part-worth utility for housing type drops fast from semi-detached house to attached house and from attached house to low-rise apartment ( $\leq 6$ floors) but tends to stabilize around low-rising apartment ( $\leq 6$ floors) and high-rising apartment ( $>6$ floors). The standard deviation results suggest that respondents' tastes differ widely if the housing type is a semi-detached house or an attached house. This result is in line with previous residential housing choice finding that residents' housing type preferences significantly vary with demographics, life events, and local housing marketing etc. (e.g., van de Vyvere et al. 1998). The estimated housing cost coefficient for carsharing-facilitating neighbourhoods rapidly decreases with increasing housing cost. Monthly housing costs of $€ 600$ and $€ 800$ have a significant high utility, while monthly housing costs of $€ 1000$ and $€ 1200$ have lower utility. The standard deviation indicates that respondents have significantly different preferences to the monthly housing costs of $€ 600$ and $€ 800$. This result is in line with the previous finding that lower housing cost is in general attractive to residents (e.g., Zondag and Pieters 2005). However, the tolerance for housing cost varies with residents' demographics, such as income (e.g., Zhou and Kockelman 2009). As for housing size, the estimated part-worth utility for housing size monotonically increases with increasing housing size. Heterogeneity depicted by the standard deviations are larger for a housing size of $40 \mathrm{~m}^{2}$ and $70 \mathrm{~m}^{2}$, but smaller for a housing size of $100 \mathrm{~m}^{2}$. This finding is as expected as residential housing choice research has found large housing is, in general, an attractive housing feature, however, the young or the senior may prefer smaller housing (e.g., Axhausen et al. 2004; Eliasson 2010). The estimated part-worth utility for housing building year increases rapidly with the housing building year becoming later, especially from a year before 1960 to a year between 1960 and 1980. A housing building year before 1960 results in a significantly large negative effect. It is consistent with the literature that housing building year before 1960 is in general disliked by residents (e.g., van de Vyvere et al. 1998; Srour et al. 2002).

(3) Effects of carsharing-facilitating neighbourhood attributes and demographics interaction

To measure the influence of specific social-demographics on the carsharing-facilitating neighbourhood attributes, the two-way interactions were entered in the model as non-random parameters. To note, to reduce estimated parameter number, only the interactions with significant coefficient were estimated in the final model. The estimated results are shown in the second subset of Table 2 . According to the results, interactions between city scale and 
housing location, city scale and housing building year, gender and housing location, gender and housing ownership, age and carsharing cost, marital status and green space density, marital status and housing type, income and housing cost have significant influence.

To start with, carsharing-facilitating neighbourhoods located in city centers have positive utility for respondents who live in big cities while having negative utility for respondents who live in medium cities. We infer that large city centers have more abundant amenities, such as historic centers, beautiful harbors, and commercial facilities etc. than medium city centers, thus more appealing to residents. In the case of the interaction between city scale and housing building year, older buildings in large cities have high utilities, while newer buildings in medium cities have high utility. One possible reason is that for large city residents, living in older housing is more economically efficient. Compared with females, males significantly prefer carsharing-facilitating neighbourhoods located in city centers and dislike housing ownership. The preferences to carsharing cost vary between age groups. Compared with young people (less than 35 years old), older people more incline to free carsharing cost in the carsharing-facilitating neighbourhoods. According to previous research, older people are less interested in carsharing but lower carsharing cost, in general, could increase residents' willingness to subscribe carsharing (e.g., Millard-Ball et al. 2005). We infer that older people need more incentive such as lower carsharing costs to accept carsharing-facilitating neighbourhoods. Couple households with children showed significant preferences for high green space density in carsharing-facilitating neighbourhoods. This result is as expected because parents lived in car-free neighbourhoods indicated high green space density is, in particular, appealing to them, because it enables their children closer to green space (e.g., Coates 2013). Besides, the couple with children households significantly prefer semi-attached housing more than single no child households. As expected, couples with children want more spacious housing for their larger households (e.g., van de Vyvere et al. 1998). Last but not least, respondents' preferences for housing cost in carsharing-facilitating neighbourhoods vary significantly with residents' income differences. Lower-income groups (less than €2400) significantly prefer a lower housing cost (€600) more than higher-income groups do. This result is in line with the previous finding that with income increase, residents' tolerance for higher housing cost increases (e.g., Zondag and Pieters 2005).

\section{(4) Main effects of demographics}

The third subset of attributes varied in the experiment are related to individual socialdemographics in terms of the city scale, gender, age, education, job type, income and marital status. They are entered in the model as non-random parameters. It shows that residents who live in a large city are more interested in living in the carsharing-facilitating neighbourhoods. This result is as expects. According to previous research, large cities are more likely to be carsharing potential markets. The reason is that residents in large cities are faced with more severe parking pressure. Besides, large cities have advanced public transportation networks which makes residents less dependent on private cars. Carsharing, serving as a supplement for public transport, helps residents in large cities fulfil a no-car life (e.g., Shaheen and Cohen 2007). The effects of the age categories suggest that respondents between 18 and 35 years old significantly intend to live in carsharing-facilitating neighbourhoods. With increasing age, utility tends to more or less monotonically decrease. The estimated effects of age are as expected in that young people are usually the early adopters of new products and innovations (e.g. Becker et al. 2017). As for the effect 
of education, low-educated respondents are less inclined to live in a carsharing-facilitating neighbourhood, which is in line with previous findings that high-educated people are more likely to be the targeted customers of carsharing related services (e.g., Ciari et al. 2016; Cartenì et al. 2016). Respondents with a part-time job are more likely to live in a carsharing-facilitating neighbourhood, while respondents who have a full-time job or do not work are less inclined to live in carsharing-facilitating neighbourhoods. We infer that since part-time workers go to office less often than full-time workers, their occasional commuting can be more easily done by carsharing or advanced public transportation provided in carsharing-facilitating neighbourhoods. However, for full-time workers, private cars are more convenient for daily use. The estimated effects of income suggest that the middleincome group who have a net monthly income between $€ 1601$ and $€ 2400$ have a higher propensity to choose a carsharing-facilitating neighbourhood. Last but not least, the effects of marital status indicate that in particular couples with children are positively inclined to live in carsharing-facilitating neighbourhoods. This result is in line with previous research which indicated the majority of car-free neighbourhood residents are young parents. Living in a car-free neighbourhood can provide their children with a better neighbourhood environment (e.g., Coates 2013).

\section{Discussion and conclusions}

Carsharing-facilitating neighbourhood is a new hybrid concept which combines carsharing, car-free transportation-residential planning, and housing attributes to promote less private car use and improve neighbourhood environment. To understand residents' preferences for such neighbourhoods, a stated-choice experiment was applied among random samples from a large national panel. A mixed logit model was estimated to assess the effects of carsharing-facilitating neighbourhood attributes, socio-demographic and their interactions on carsharing-facilitating neighbourhood utilities.

According to the results, low carsharing cost, little carsharing booking time, less than 5 min walking or 6-10 min walking to shared-car parking, high green space density, large housing size, low housing costs and housing built after 1960 in general increase carsharing-facilitating neighbourhood utility. These results indicated that good carsharing services can efficiently improve the appealing of carsharing-facilitating neighbourhood. However, one must notice fundamental housing attributes still play dominant roles in residents' carsharing-facilitating neighbourhood choices. Though traditional sustainable transportationresidential planning attributes have limited influence on residents' choices, these attributes cannot be neglected by developers because they provide basic conditions for car-reduced developments. Last but least, the influence of residents' demographics on the carsharingfacilitating neighbourhood utility indicated residents who live in large cities, living with partners and children, having higher education, a part-time job and net monthly income between $€ 1601$ and $€ 2400$ are more willing to live in carsharing-facilitating neighbourhoods. These results provide information about targeted carsharing-facilitating neighbourhood customer groups. By referring the local residents' demographics profiles, governments, real estate agents or carsharing operators could estimate and locate potential marketing, thus select candidate sites for carsharing-facilitating neighbourhoods.

Several problems remain for future research. In case of the stated choice experimental design, certain complex contexts like private car ownership restrictions (no-car contract, lottery private parking allocation etc.) and integrated green transportation strategy etc. were 
simplified to private parking availability and public transport accessibility. As a pioneered exploratory research, it greatly simplifies the experimental design and helped to focus on principal influence factors. However, it may also weaken or conceal the effects of these elaborated context elements. To clarify these effects, specialized researches are needed in the future. Besides, considering the carsharing-facilitating neighbourhood choice is often a household decision, one should elaborate on the approach and model into a model of household decision making. It will also be of interest to extend carsharing conception to commercial zone or office area to citizens' preferences to them, thus combine with our current research results, we can further develop urban land use carsharing system strategy. We intend to report such research in future publications.

Open Access This article is licensed under a Creative Commons Attribution 4.0 International License, which permits use, sharing, adaptation, distribution and reproduction in any medium or format, as long as you give appropriate credit to the original author(s) and the source, provide a link to the Creative Commons licence, and indicate if changes were made. The images or other third party material in this article are included in the article's Creative Commons licence, unless indicated otherwise in a credit line to the material. If material is not included in the article's Creative Commons licence and your intended use is not permitted by statutory regulation or exceeds the permitted use, you will need to obtain permission directly from the copyright holder. To view a copy of this licence, visit http://creativecommons.org/licenses/by/4.0/.

\section{References}

Agatz, N., Erera, A., Savelsbergh, M., \& Wang, X. (2012). Optimization for dynamic ride-sharing: A review. European Journal of Operational Research, 223(2), 295-303.

Axhausen, K. W., Konig, A., Scott, D. M., \& Jurgens, C. (2004). Locations, commitments and activity spaces. Human Behavior and Traffic Networks, 1, 205-230.

Banister, D., Akerman, J., Nijkamp, P., Stead, D., Dreborg, K., Steen, P., et al. (2000). European transport policy and sustainable mobility. Milton Park: Taylor \& Francis.

Becker, H., Ciari, F., \& Axhausen, K. W. (2017). Comparing car-Sharing schemes in switzerland: User groups and usage patterns. Transportation Research Part A: Policy and Practice, 97, 17-29.

Bhat, C. R. (2001). Quasi-random maximum simulated likelihood estimation of the mixed multinomial logit model. Transportation Research Part B: Methodological, 35(7), 677-693.

Biswas, S., Chandra, S., \& Ghosh, I. (2017). Effects of on-street parking in urban context: A critical review. Transportation in developing economies., 3(1), 10.

Bock, G. W., Zmud, R. W., Kim, Y. G., \& Lee, J. N. (2005). Behavioral intention formation in knowledge sharing: Examining the roles of extrinsic motivators, social-psychological forces, and organizational climate. MIS Quarterly, 1, 87-111.

Buse, A. (1982). The likelihood ratio, Wald, and Lagrange multiplier tests: An expository note. The American Statistician, 36(3a), 153-157.

Cartenì, A., Cascetta, E., \& de Luca, S. (2016). A random utility model for park \& carsharing services and the pure preference for electric vehicles. Transport Policy, 48, 49-59.

Chatman, D. G. (2009). Residential choice, the built environment, and nonwork travel: evidence using new data and methods. Environment and planning A., 41(5), 1072-1089.

Chen, J., Chen, C., \& Timmermans, H. (2008). Accessibility trade-offs in household residential location decisions. Transportation Research Record, 2077(1), 71-79.

Ciari, F., Weis, C., \& Balac, M. (2016). Evaluating the influence of carsharing stations? Location on potential membership: A Swiss case study. EURO Journal on Transportation and Logistics, 5(3), 345-369.

Coates, G. J. (2013). The sustainable urban district of vauban in Freiburg, Germany. International Journal of Design \& Nature and Ecodynamics, 8(4), 265-286.

De Palma, A., Motamedi, K., Picard, N., \& Waddell, P. (2005). A model of residential location choice with endogenous housing prices and traffic for the Paris region. European Transport, 31, 67-82.

De Palma, A., Picard, N., \& Waddell, P. (2007). Discrete choice models with capacity constraints: An empirical analysis of the housing market of the greater Paris region. Journal of Urban Economics, 62(2), 204-230. 
De Vos, J., Derudder, B., Van Acker, V., \& Witlox, F. (2012). Reducing car use: changing attitudes or relocating? The influence of residential dissonance on travel behavior. Journal of Transport Geography, 22, 1-9.

Dieleman, F. (1996). Households and housing: Choice and outcomes in the housing market. London: Routledge.

Dowling, R., \& Kent, J. (2015). Practice and public-private partnerships in sustainable transport governance: The case of car sharing in Sydney, Australia. Transport policy, 40, 58-64.

Eliasson, J. (2010). The influence of accessibility on residential location (pp. 137-164). Spinger: Residential Location Choice. Berlin.

European Commission. (2017). Sustainable urban mobility: European policy, practice and solutions. European Union: Report.

Foletta, N., \& Field, S. (2011). Europe's vibrant new low car (bon) communities. New York: ITDP.

Greene, W. H. (2001). Fixed and random effects in nonlinear models. NYU Working Paper No. EC-01-01.

Guo, J. Y., \& Bhat, C. R. (2007). Operationalizing the concept of neighbourhood: Application to residential location choice analysis. Journal of Transport Geography, 15(1), 31-45.

Habib, M., \& Miller, E. (2009). Reference-dependent residential location choice model within a relocation context. Transportation Research Record, 2133(1), 92-99.

Hensher, D. A., Rose, J. M., \& Greene, W. H. (2005). Applied choice analysis: a primer. Cambridge: Cambridge University Press.

Herranz, A. (2020). Merwede, the Dutch neighbourhood where there will be one shared car for every 3 households, Tomorrow. MAG by tomorrow. City. https://www.fastcompany.com/90457158/in-thisnew-dutch-neighbourhood-there-will-be-1-shared-car-for-every-3-households. 25 March, 2020.

Joo, J. H. (2017). Motives for participating in sharing economy: Intentions to use car Sharing services. Journal of Distribution Science, 15(2), 21-26.

Kato, H., Inagi, A., \& Igo, T. (2013). Potential choices of travel mode including carsharing and carsharing membership: Evidences from four japanese cities. Journal of the Eastern Asia Society for Transportation Studies, 10, 630-646.

KE, Train. (2003). Discrete choice methods with simulation. Cambridge: Cambridge University Press.

Kim, J., Pagliara, F., \& Preston, J. (2005). The intention to move and residential location choice behaviour. Urban Studies, 42(9), 1621-1636.

Kim, J., Rasouli, S., \& Timmermans, H. (2017). Satisfaction and uncertainty in car-sharing decisions: An integration of hybrid choice and random regret-based models. Transportation Research Part A: Policy and Practice. Elsevier Ltd, 95, 13-33.

Kopp, J., Gerike, R., \& Axhausen, K. W. (2015). Do sharing people behave differently? An empirical evaluation of the distinctive mobility pattern of free-floating car-sharing members. Transportation, 42(3), 449-469.

Kushner, J. A. (2005). Car-Free housing developments: Towards sustainable smart growth and urban regeneration through car-free zoning, car-free redevelopment, pedestrian improvement districts, and new urbanism. UCLA Journal of Environmental Law and Policy, 23(1), 1.

Loose, W. (2015). The state of European car-sharing. Bundesverband Carsharing e. V., Final repo (p. 84). http://www.slideshare.net/AtomicoVentures/the-state-of-european-tech.

Louviere, J., \& Timmermans, H. (1990). Stated preference and choice models applied to recreation research: a review. Leisure Sciences, 12(1), 9-32.

Louviere, J. J., Hensher, D. A., \& Swait, J. D. (2000). Stated choice methods: Analysis and applications. Cambridge: Cambridge University.

Martens, K., Sierzchula, W., \& Pasman, S. (2015). Broadening the market for carshare? Results of a pilot project in the Netherlands. World Tranport Policy and Practice, 21(1), 17-29.

McFadden, D. (1973). Conditional logit analysis of qualitative choice behavior. Frontiers in Econometrics (pp. 105-142). New York: Academic Press.

Melia, S. (2010). Carfree, low car - what's the difference? Paper presented at European Transport Conference.

Millard-Ball, A., Murray, G., Schure, J. T., Fox, C., \& Burkhardt, J. (2005). Car-sharing: Where and how it succeeds. Report, Transportation Research Board: Washington, DC.

Morris, D., Enoch, M., Pitfield, D., \& Ison, S. (2009). Car-free development through UK community travel plans. Proceedings of the Institution of Civil Engineers : Urban Design and Planning, 162(DP1), 19-27.

Newman, P., Kosonen, L., \& Kenworthy, J. (2016). Theory of urban fabrics: Planning the walking, transit/public transport and automobile/motor car cities for reduced car dependency. Town Planning Review, 87(4), 429-458. 
Nijland, H., \& van Meerkerk, J. (2017). Mobility and environmental impacts of car sharing in the Netherlands. Environmental Innovation and Societal Transitions (pp. 84-91). New York: Elsevier.

Olanrewaju, A., \& Woon, T. C. (2017). An exploration of determinants of affordable housing choice. International Journal of Housing Markets and Analysis, 10(5), 703-723.

Pinjari, A. R., Bhat, C. R., \& Hensher, D. A. (2009). Residential self-selection effects in an activity time-use behavior model. Transportation Research Part B: Methodological, 43(7), 729-748.

Rao, V. R. (2014). Applied conjoint analysis. New York: Springer; Mar.

Rodier, C., \& Shaheen, A. S. (2003). Carsharing and carfree housing: predicted travel, emission, and economic benefits: A case study of the Sacramento, California Region. In: Transportation Research Board Annual Meeting.

Schirmer, P. M., Van Eggermond, M. A. B., \& Axhausen, K. W. (2014). The role of location in residential location choice models: a review of literature. Journal of Transport and Land Use, 7(2), 3-21.

Shaheen, S. A., \& Cohen, A. P. (2007). Worldwide carsharing growth: An international comparison. Transportation Research Record, 1992(1), 81-89.

Srour, I. M., Kockelman, K. M., \& Dunn, T. P. (2002). Accessibility indices: Connection to residential land prices and location choices. Transportation Research Record, 1805(1), 25-34.

Tu, Y., Li, P., \& Qiu, L. (2017). Housing search and housing choice in urban China. Urban Studies, 54(8), 1851-1866.

Van de Vyvere, Y., Oppewal, H., \& Timmermans, H. (1998). The validity of hierarchical information integration choice experiments to model residential preference and choice. Geographical Analysis, 30(3), 254-272.

Waddell, P. (2006). Reconciling household residential location choices and neighborhood dynamics. Under revision, sociological methods and research.

Wang, N., \& Yan, R. (2016). Research on consumers' use willingness and opinions of electric vehicle sharing : An empirical study in shanghai. Sustainability, 8(1), 7.

Weisbrod, G., Lerman, S. R., \& Ben-Akiva, M. (1980). Tradeoffs in residential location decisions: Transportation versus other factors. Transport Policy and Decision Making, 1(1), 13-26.

Wiersma, J., Bertolini, L., \& Straatemeier, T. (2016). How does the spatial context shape conditions for car dependency? An analysis of the differences between and within regions in the Netherlands. Journal of Transport and Land Use, 9(3), 35-55.

Zhou, B., \& Kockelman, K. M. (2009). Microsimulation of residential land development and household location choices: Bidding for land in Austin. Texas. Transportation Research Record, 2077(1), 106-112.

Zondag, B., \& Pieters, M. (2005). Influence of accessibility on residential location choice. Transportation Research Record, 1902(1), 63-70.

Publisher's Note Springer Nature remains neutral with regard to jurisdictional claims in published maps and institutional affiliations. 